\title{
Madelung and pseudo Madelung deformities-Pictorial essay
}

\author{
Subbarao Kakarla ${ }^{1, *}$
}

${ }^{1}$ KIMS Foundation and Research Centre, Minister Road, Secunderabad - 500003, Telangana, India

\begin{abstract}
Madelung deformity of the wrist is a rare disorder. This often presents with a short forearm. This entity is also known as mesomelic dwarfism of Leri and Weill. Female preponderance is noted and is autosomal dominant. This may be bilateral in 50-66\% of patients. Clinical diagnosis is often suggested but radiological confirmation is essential. Pseudo Madelung deformity simulates Madelung deformity except in the former there is negative ulnar variance with the distal articular surface of the ulna articulating with the medial cortex of the distal radial metaphysis. It also includes 'reverse Madelung deformity'. Three major causes of deformity exist: 1. Idiopathic, 2. Hereditary, 3. Acquired. Radiologically, shortening and bowing of radius is noted with dysgenesis of the distal radial epiphysis. Subluxation of the distal ulna is noted as well as dislocation at the elbow. Surgical correction is the only treatment with genetic consultation to the parents.
\end{abstract}

Keywords: Madelung deformity; short radius; dislocation of the distal ulna; proximal radius; Leri weill; dyschondrosteosis

*Corresponding author: Prof. Kakarla Subbarao, MS, D.Sc. (HON), FRCR, FACR, FICP, FSASMA, FCCP, FICR, FCGP, Chairman, KIMS Foundation and Research Centre, Minister Road, Secunderabad 500003, Telangana, India. Email: subbaraokakarla25@gmail.com

Received 13 September 2018; Revised 22 November 2018; Accepted 30 November 2018; Published 10 December 2018

Citation: Kakarla S. Madelung and pseudo Madelung deformitiesPictorial essay. J Med Sci Res. 2019; 7(1):1-6. DOI: http://dx.doi. org/10.17727/JMSR.2019/7-1

Copyright: (C) 2019 Kakarla S et al. Published by KIMS Foundation and Research Center. This is an open-access article distributed under the terms of the Creative Commons Attribution License, which permits unrestricted use, distribution, and reproduction in any medium, provided the original author and source are credited.

\section{Introduction}

When the disorder is bilateral and is familial, it is categorised under mesomelic dwarfism. Short tibia and fibula may also be associated. Originally described by the German surgeon Otto Madelung in 1879. Leri and Weill have elaborated this entity later in 1929 [1]. It has not received much attention, until the later decades of $20^{\text {th }}$ century [2, 3]. Radiological findings are described by Kozlowski et al., in the name of dyschondrosteosis in 1971 [4]. Clinically, adolescent girls present with pain in the wrist and decreased range of motion. Madelung deformity is often used to describe a variety of conditions in the wrist marked by premature fusion of the distal physis of the radius, with consequent deformity of the distal ulna and wrist. These include post-traumatic and infective deformities, dysplasias, Turner syndrome and idiopathic. 


\section{Discussion}

Leri-Weill dyschondrosteosis (LWD) [1] is a skeletal dysplasia characterized by short stature and an abnormality of the wrist bones called Madelung deformity. Short stature is present from birth due to shortening of the long bones in the legs. Madelung deformity typically develops during midto-late childhood and may progress during puberty. Persons with this condition often experience pain in their wrists or arms. The severity of LWD varies among affected individuals, although the signs and symptoms of this condition are generally more severe in females. Other features of LWD can include increased muscle size, bowing of the tibia, elbow abnormalities, scoliosis, and high-arched palate. Intelligence is not affected in this disorder. Most cases of LWD are caused by mutations in or near the shox gene. The cause of the disorder remains unknown in those cases not related to the shox gene. LWD follows a pseudoautosomal dominant pattern of inheritance [1-4]. Madelung deformity is an epiphyseal growth plate disturbance characterised by dorsal and radial bowing of the radius with resultant cosmetic effects and decreased grip strength. The common mechanism for all causes of Madelung deformity is partial closure, or failure of development of the ulnar side of the distal radial growth plate. There is an arrest of epiphyseal growth of the medial and volar (anterior) portions of the distal radius. This leads to shortening of the radius and relative overgrowth of the ulna. It is associated with several disorders including congenital, acquired. These are listed in Table 1. Pseudo Madelung deformity simulates Madelung deformity except in the former there is negative ulnar variance with the distal articular surface of the ulna articulating with the medial cortex of the distal radial metaphysis.

\section{Associated disorders - Madelung and pseudo Madelung}

Table 1: Congenital.

\begin{tabular}{rl|}
$>$ & Leri-Weill syndrome \\
$>$ & Turner syndrome \\
$>$ & Nail-patella syndrome \\
$>$ & Hereditary, multiple exostosis \\
$>$ & Achondroplasia \\
$>\quad$ Multiple epiphyseal dysplasias \\
$>\quad$ Mucopolysaccharidoses \\
& (Hurler and Morquio syndromes) \\
\hline
\end{tabular}

\section{Acquired}

$>$ Traumatic

$>$ Infective

In Leri-Weill syndrome, Madelung deformity presents as a spectrum of findings. It may affect the entire radius or it may affect only the distal radius. Extremities with involvement of the entire radius have a shorter radius and ulna, decreased height, and a more severe deformity of the extremities than with involvement of only the distal radius [5-7]. The radiological findings are summarized in Table 2. Radiological findings of Madelung (Figure 1).

Table 2: The deformity is characterised by:

$>$ Dorsal and radial bowing of the radius

$>$ Exaggerated palmar (up to $35^{\circ}$ ) and ulnar tilt (up to $60^{\circ}$ ) of the radiocarpal articulation

$>$ Failure of ossification of the ulnar side of the distal radial epiphysis

$>$ Exaggerated radial inclination

$>$ Decreased carpal angle below $118^{\circ}$; normal from $118^{\circ}$ to $139^{\circ}$

$>$ Carpal subluxation in a palmar and ulnar direction

$>$ Lunate is gradually forced to the apex of the V-shaped radioulnaocarpal joint

$>$ "V-shaped" proximal carpal row $=$ herniated proximal carpal row

$>$ Dorsal subluxation of the distal ulna

$>$ Elongation of the ulna with positive ulnar variance

$>$ Wedging of the carpus between the radius and ulna

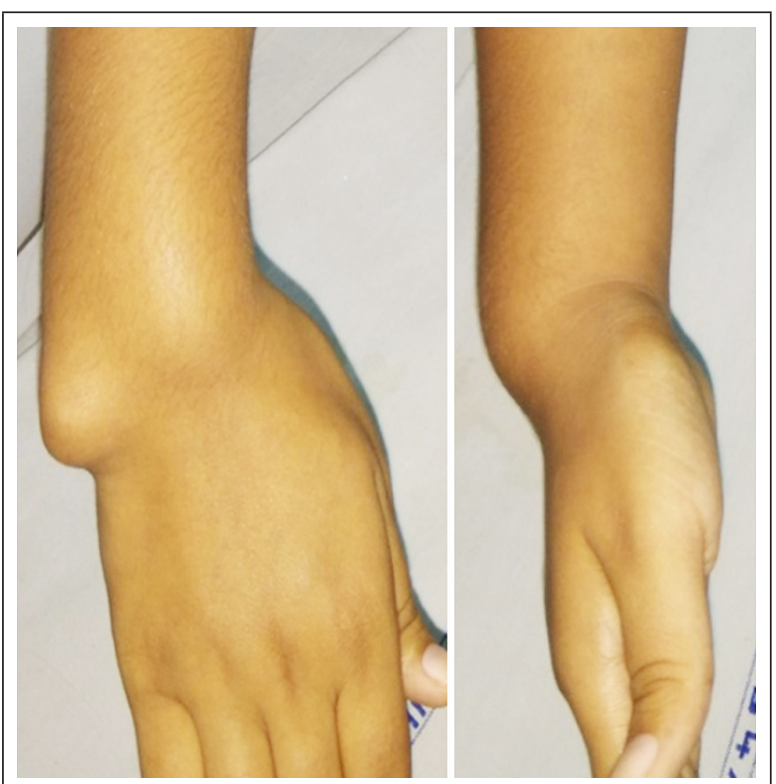

Figure 1a: Madelung deformity, clinical picture. 

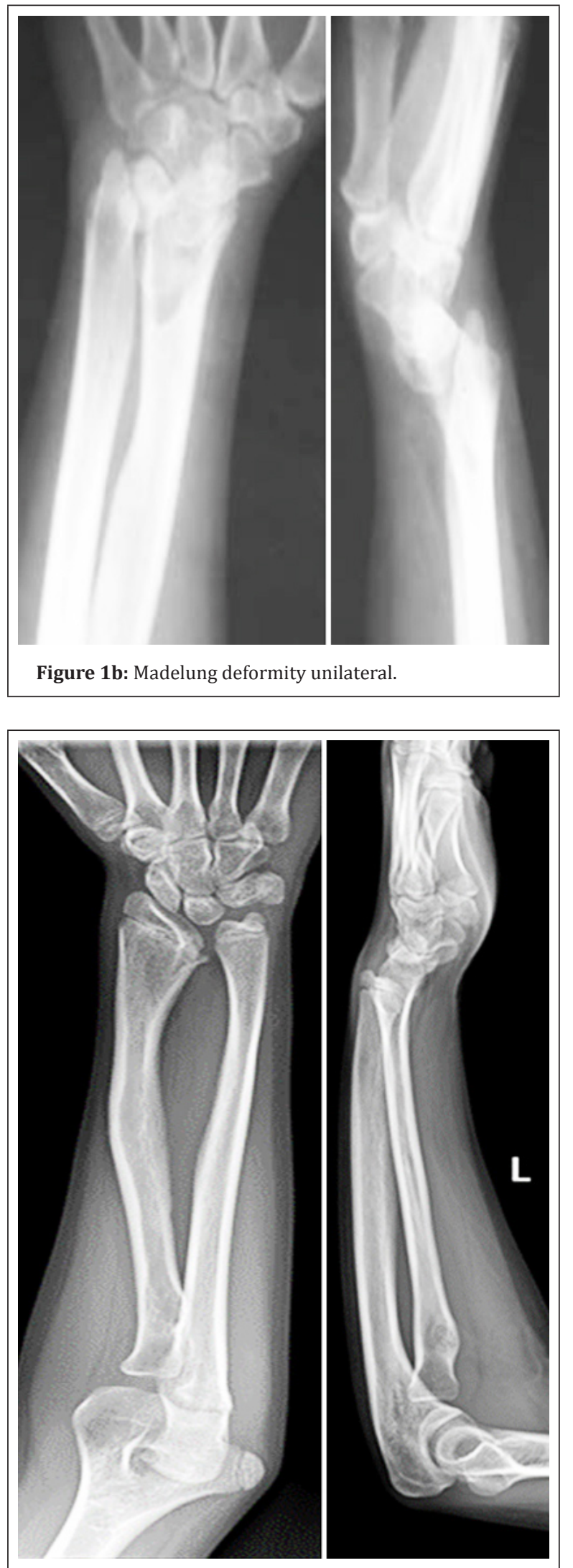

Figure 1c: 11-year-old female, Madelung (Note: dislocation of the head of the radius).

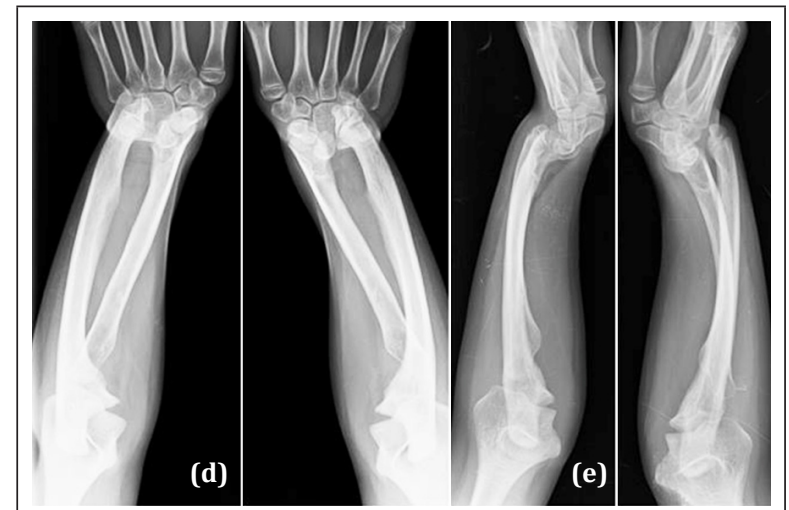

Figure 1d, e: Bilateral Madelung deformity; (d) PA view, (e) Lateral.

Pseudo Madelung - radiological characteristics are listed in Table 3 (Figure 2).

Table 3: Absence of:

\begin{tabular}{|c|c|}
\hline$>$ & Positive ulnar variance \\
\hline$>$ & Dorsal subluxation of the distal ulna \\
\hline$>$ & Wedging of the carpus between the radius and ulna \\
\hline$>$ & Dorsal and radial bowing of the radius \\
\hline$>$ & $\begin{array}{l}\text { Failure of ossification of the ulnar side of the distal radial } \\
\text { epiphysis }\end{array}$ \\
\hline$>$ & $\begin{array}{l}\text { "V-shaped" proximal carpal row (herniated proximal } \\
\text { carpal row) }\end{array}$ \\
\hline
\end{tabular}

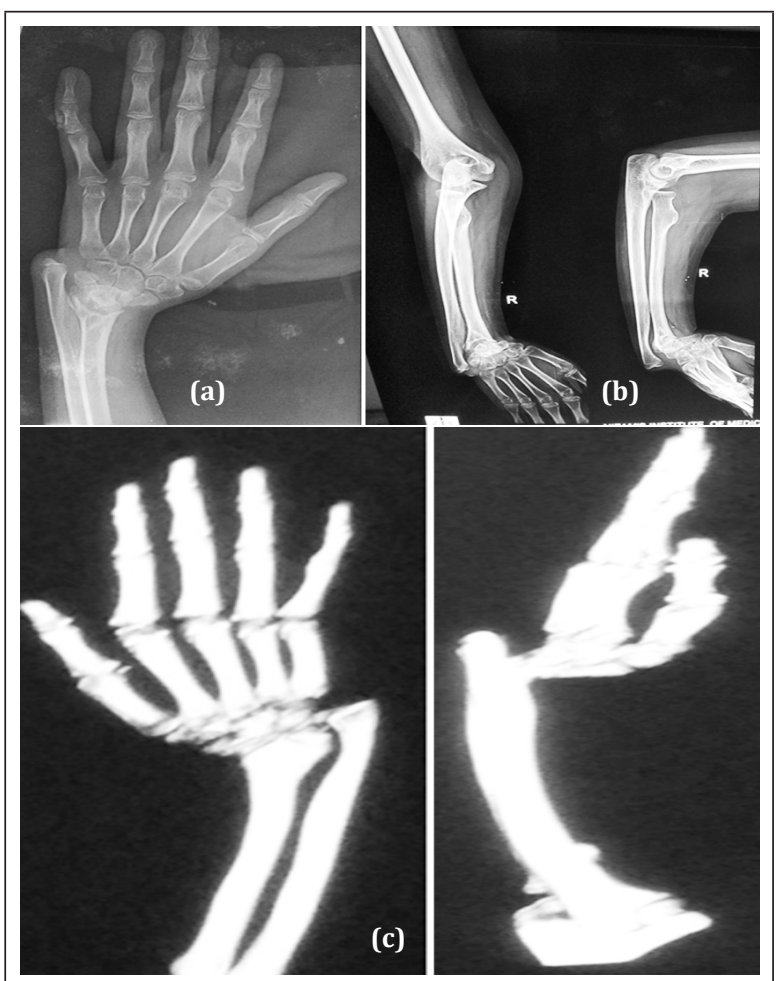

Figure 2a, b, c: Madelung with elbow deformity showing dislocation of head of radius; (c) 3D CT. 
Pseudo Madelung deformity is noted in achondroplasia which also shows dumbbell shaped bones which are short with cup-shaped articular margins (Figure 3a, b).

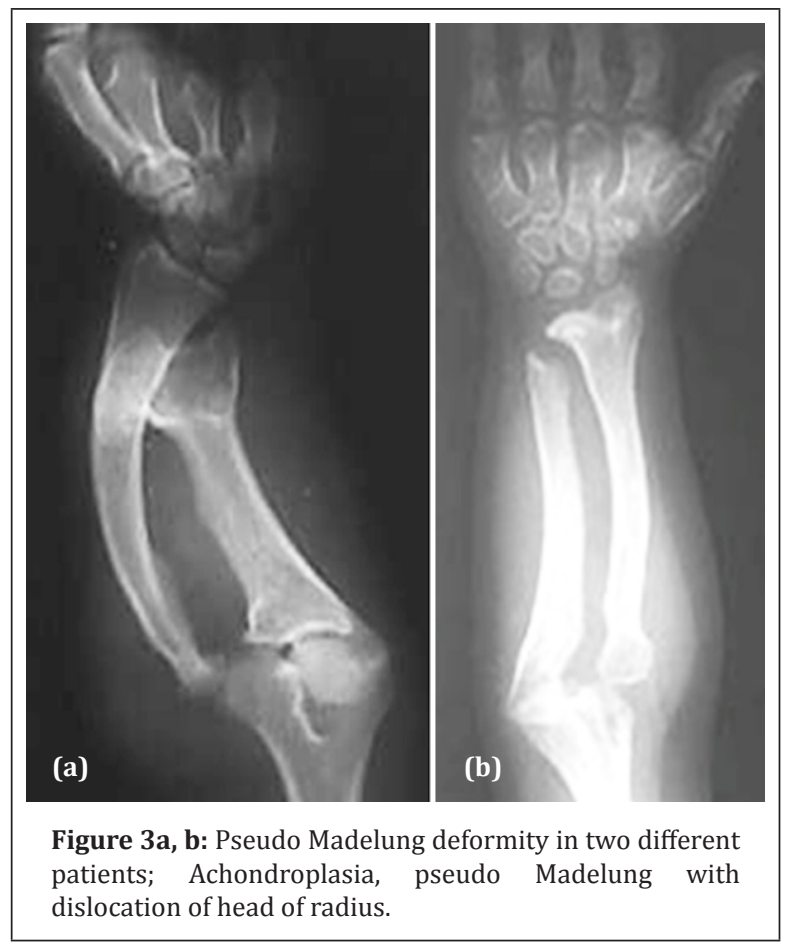

Pseudo Madelung deformity is also noted in mucopolysaccharidoses specially in Morquio and Hurler entities. Brachydactyly and bullet shaped proximal metacarpals are the character features (Figure 3c, d).

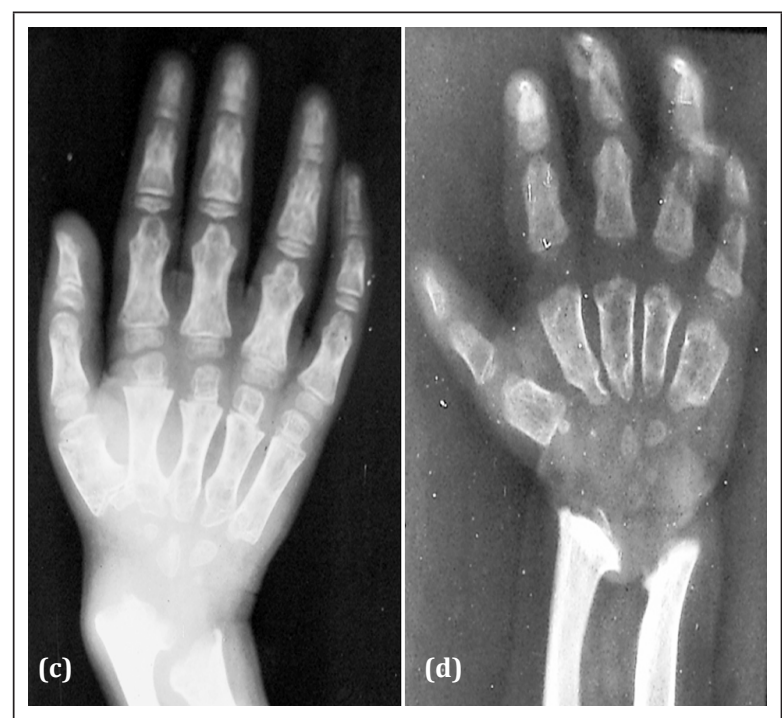

Figure 3c, d: Pseudo Madelung; (c) Hurler, (d) Morquio Mucopolysaccharidosis.
Turner syndrome: In addition to pseudo Madelung deformity short fourth metacarpal is an associated feature [7] (Figure 4a, b).

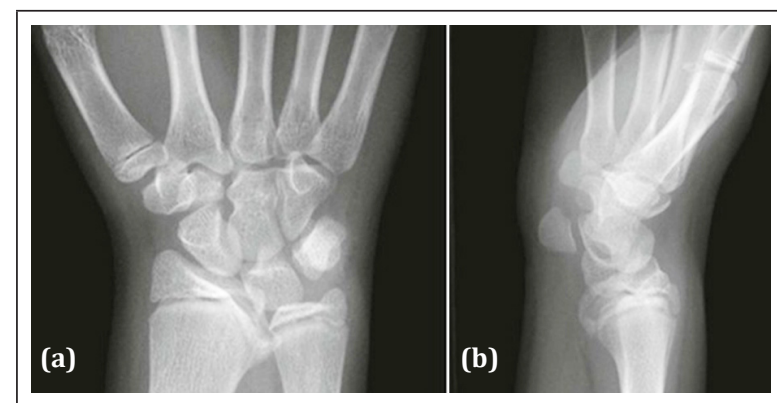

Figure 4a, b: Pseudo Madelung; Turner syndrome - Small carpal angle.

In Hereditary multiple exostosis (Osteochondromata), pseudo Madelung deformity is noted (Figure $5 a, b, c)$. The distal end of the ulna shows bayonet deformity.

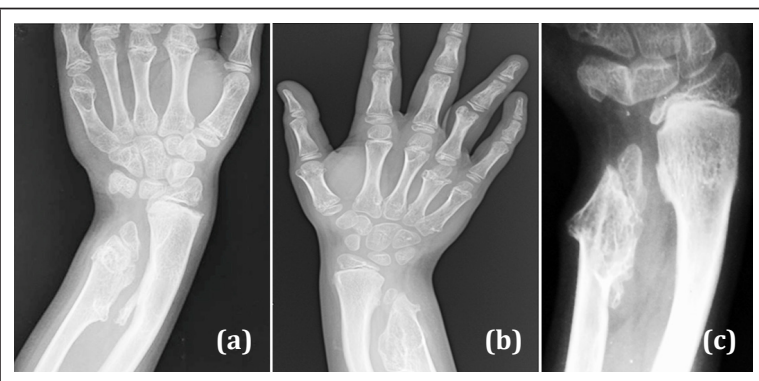

Figure 5a, b, c: Pseudomadelung due to hereditary multiple exostoses. Autosomal dominent with incomplete penetrance in females. Likely defect in tumor suppressor genes EXT in chromosome 8, 11, 1; (c) Bayonet deformity.

Pseudo Madelung deformity is also noted in Oller's dyschondroplasia, where multiple enchondromata of metacarpals and phalanges are noted (Figure $5 \mathrm{~d}$, $e, f)$.

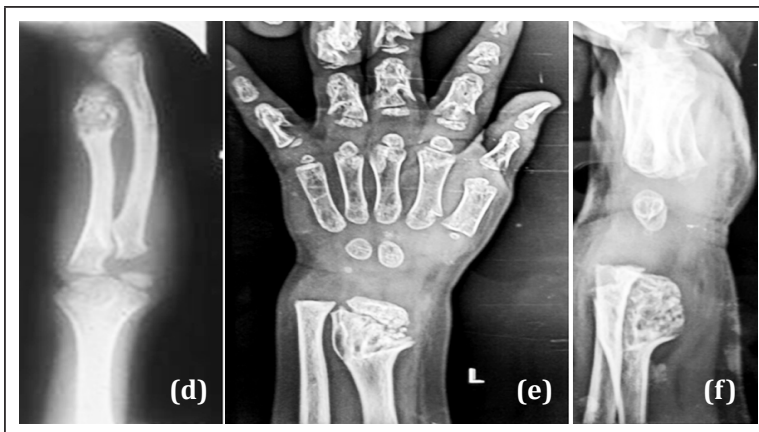

Figure 5d, e, f: Pseudo Madelung in Ollier's dyschondroplasia. 
In trauma to the wrists pseudo Madelung deformity in various degrees is noted. In Colles fracture, 'Reverse Madelung' appearing like a dinner fork is noted [8, 9] (Figure 6).

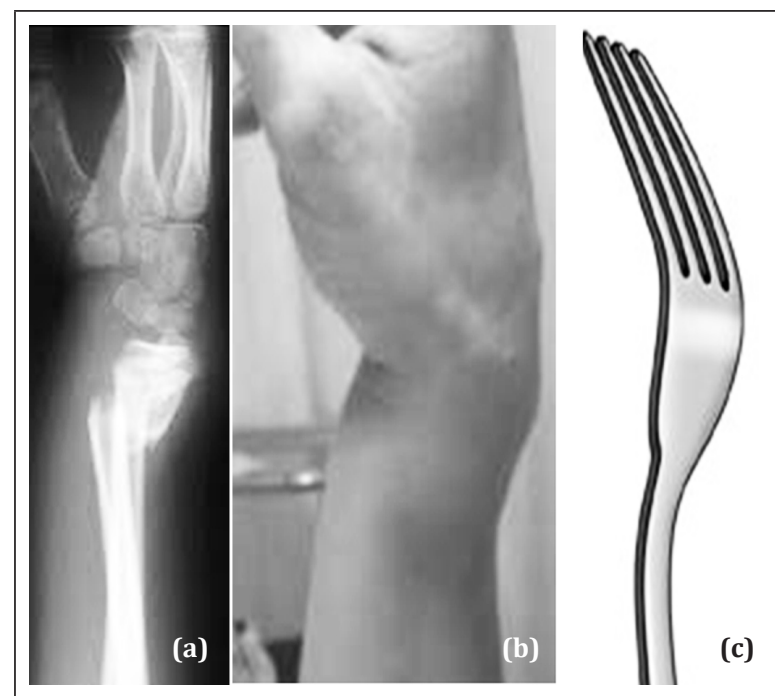

Figure 6a, b, c: Pseudo Madelung; (a) Colles fracture with dinner fork deformity, (b) Clinical, (c) Dinner fork.

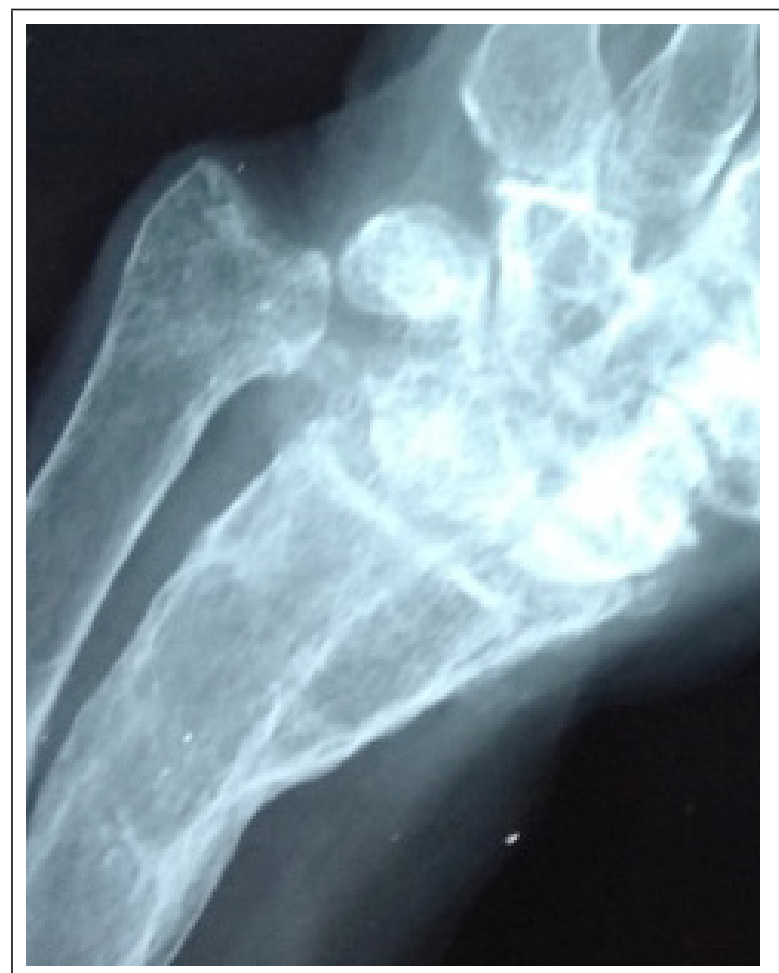

Figure 6d: Post traumatic Madelung deformity.

In infections, involving the wrist bones either by pyogenic or leprotic bacilli, particularly in chronic osteomyelitis. Madelung deformity may be seen [10] (Figure 7).

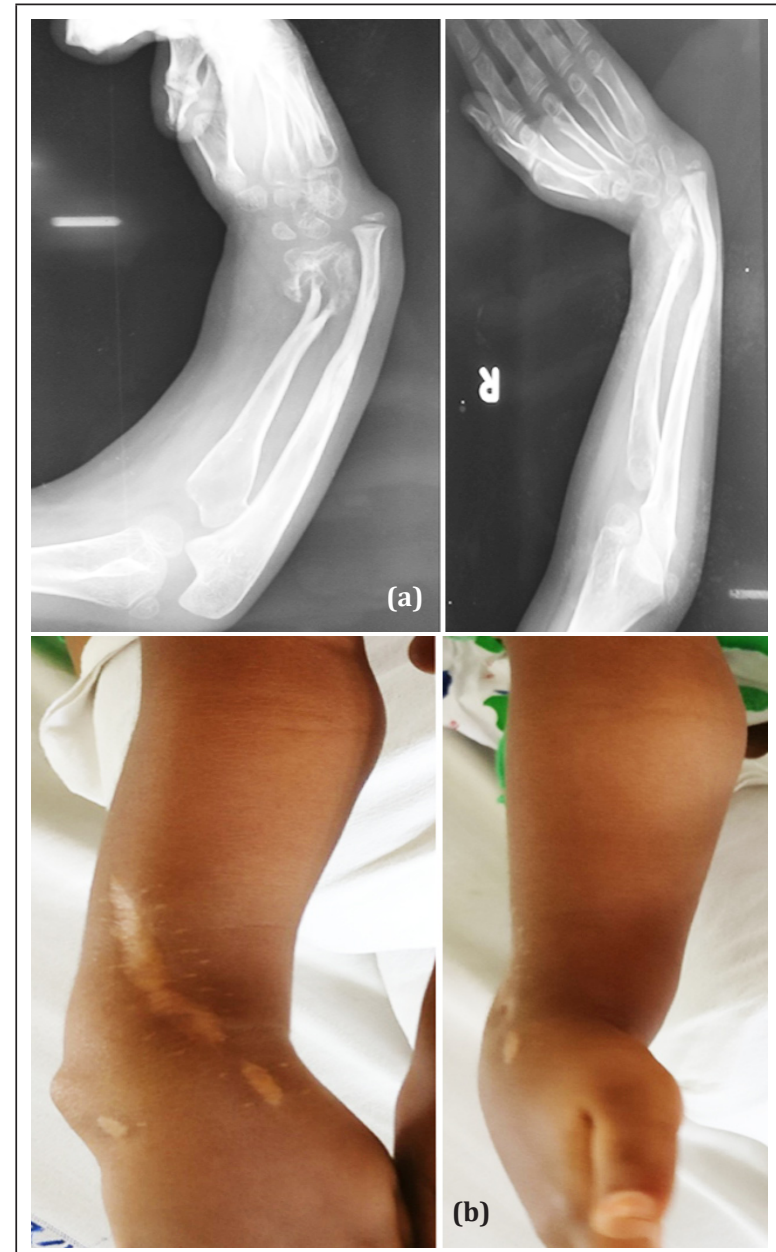

Figure 7a, b: Madelung deformity, sequelae of old fracture.

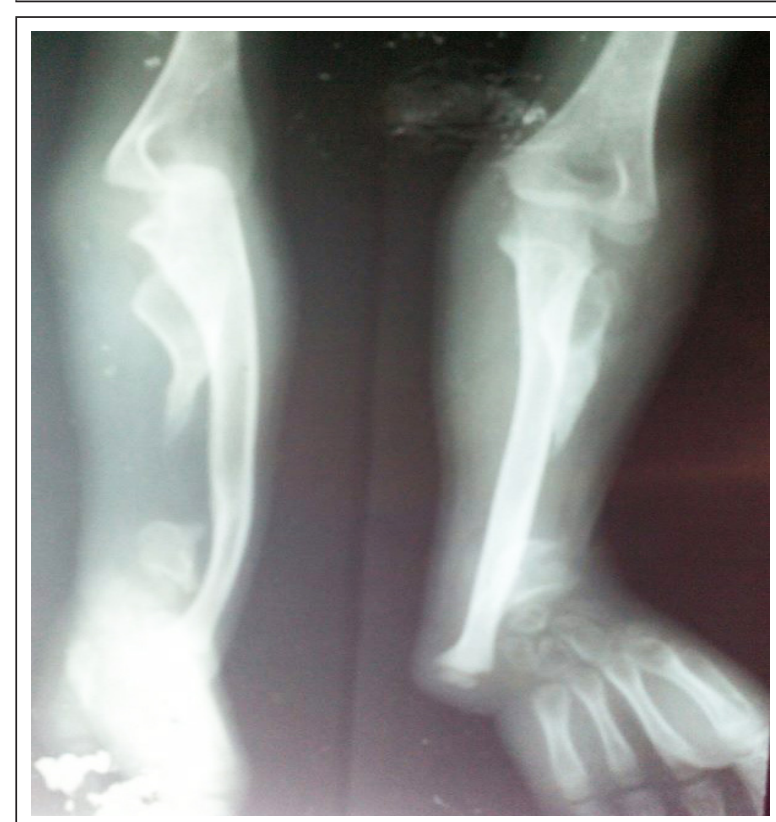

Figure 7c: Madelung deformity due to chronic osteomyelitis of the radius. 


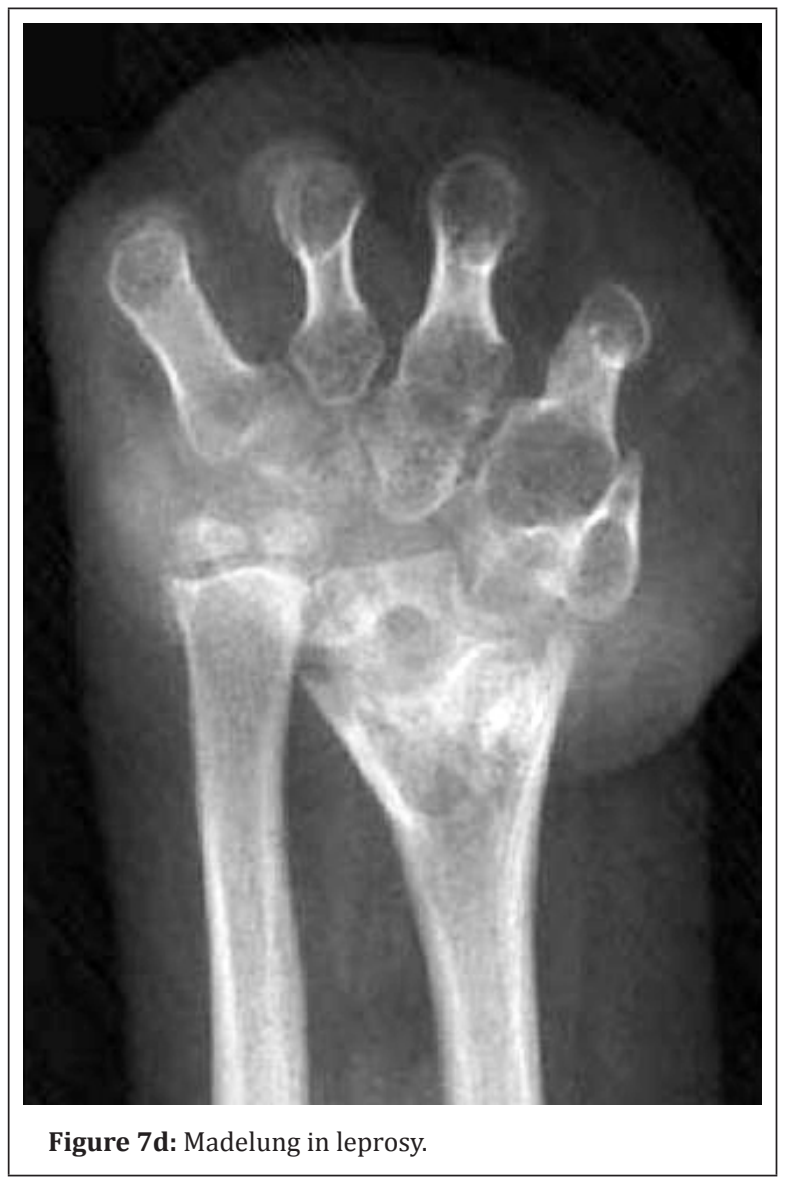

\section{Conclusion}

Madelung deformity of the wrists is rarely encountered in clinical practice but has definite radiological appearances. Apart from idiopathic causes many other etiological factors are involved in congenital and dysostotic entities. Pseudo Madelung deformity is encountered in endocrinal, dysplastic and neoplastic conditions. Pseudo/reverse Madelung deformity is more common and the radiological characteristics vary, which are described with the help of several illustrations.

\section{Acknowledgements}

NIMS, KIMS, KREST Museum Hyderabad, India.

\section{Conflicts of interest}

Author declares no conflicts of interest.

\section{References}

[1] Leri A, Weill JA. Une affection congénitale et symétrique du développement osseux. La dyschondrostéose. Bull. Mem. Soc. Med. Hop. Paris. 1929; 53:1491-1494.

[2] Kozlowski K, Zychowicz C. Dyschondrosteosis. Acta Radiol Diagn (Stockh) 1971; 11:459-466.
[3] Silverman FN. Mesomelic dwarfism. In: Kaufmann HJ (ed) Progress in Pediatric Radiology, Karger, Basel, 1973; 4:546.

[4] Weerakkody Y, Gaillard AF. Madelung deformity: Musculoskeletal, Paediatrics, Radiopaedia. Accessed from: https://radiopaedia.org/articles/madelung-deformity.

[5] Lamberti PM, Light TR. Madelung Deformity. Accessed from: https://emedicine.medscape.com/article/1260002overview.

[6] Zebala LP, Manske PR, Goldfarb CA. Madelung's deformity: a spectrum of presentation. J Hand Surg Am. 2007; 32(9):1393-1401.

[7] Binder G, Fritsch H, Schweizer R, Ranke MB. Radiological signs of Leri-Weill dyschondrosteosis in Turner syndrome. Horm Res. 2001; 55(2):71-76.

[8] Morgan AM. Pseudo-madelung deformity (post trauma); Radiopaedia. Accessed from: https://radiopaedia.org/ cases/pseudo-madelung-deformity-post-trauma.

[9] Moazami-Goudarzi Y, Hertel P, Khodadadyan C. True Madelung's deformity and traumatic pseudo-Madelung. An exemplary differential diagnosis of the deformity in the forearm of children; Aktuelle Traumatol. 1991; 21(6):279284.

[10] Huguet S, Leheup B, Aslan M, Muller F, Dautel G et al. Radiological and clinical analysis of Madelung's deformity in children. Orthop Traumatol Surg Res. 2014; $100(6$ Suppl):S349-S352. 